Journal of Fluid Mechanics, vol. 928, A29, doi:10.1017/jfm.2021.825

\title{
Self-similar fault slip in response to fluid injection
}

\author{
Robert C. Viesca ${ }^{1} \dagger$ \\ ${ }^{1}$ Department of Civil and Environmental Engineering, Tufts University, Medford, MA 02155, USA
}

(Received 31 May 2021; revised 7 Sept. 2021; published 12 Oct. 2021)

There is scientific and industrial interest in understanding how geologic faults respond to transient sources of fluid. Natural and artificial sources can elevate pore fluid pressure on the fault frictional interface, which may induce slip. We consider a simple boundary value problem to provide an elementary model of the physical process and to provide a benchmark for numerical solution procedures. We examine the slip of a fault that is an interface of two elastic half-spaces. Injection is modeled as a line source at constant pressure and fluid pressure is assumed to diffuse along the interface. The resulting problem is an integrodifferential equation governing fault slip, which has a single dimensionless parameter. The expansion of slip is self-similar and the rupture front propagates at a factor $\lambda$ of the diffusive lengthscale $\sqrt{\alpha t}$. We identify two asymptotic regimes corresponding to $\lambda$ being small or large and perform a perturbation expansion in each limit. For large $\lambda$, in the regime of a socalled critically stressed fault, a boundary layer emerges on the diffusive lengthscale, which lags far behind the rupture front. We demonstrate higher-order matched asymptotics for the integro-differential equation, and in doing so, we derive a multipole expansion to capture successive orders of influence on the outer problem for fault slip for a driving force that is small relative to the crack dimensions. Asymptotic expansions are compared to accurate numerical solutions to the full problem, which are tabulated to high precision.

\section{Introduction}

The coupling of fluid flow and fracture of an elastic medium has an extensive history in the context of open-mode fractures, specifically hydraulic fracture, in which an injected fluid drives crack opening. Early work presumed laminar flow of the fluid in a planar crack (Khristianovic and Zheltov 1955; Barenblatt 1956) leading to similarity solutions and crack-tip asymptotics (Spence and Sharp 1985; Desroches et al. 1994) that depart from the square-root asymptotic behavior of classical linear elastic fracture mechanics. Further developments include explicit consideration of fluid leak-off into the elastic medium (Lenoach 1995; Adachi and Detournay 2008), the possibility of fluid lagging behind the crack tip (Garagash and Detournay 2000), and turbulent flow (Lister 1990b; Tsai and Rice 2010), with Detournay (2016) providing a more complete review of related progress. In addition to applications to oil and gas extraction from subsurface reservoirs, such models have also been widely applied to magmatic intrusions (Lister 1990a,b; Rubin 1995; Bunger and Cruden 2011; Michaut 2011).

The interplay between fluid flow and elasticity has received renewed interest in a

$\dagger$ Email address for correspondence: robert.viesca@tufts.edu 
comparable problem of fluid-driven delamination of a thin elastic sheet from a rigid substrate. In this problem, the sheet's elastic response is represented by classical beam theory, such that elastic interactions are local and solutions are found in a relatively straightforward manner. In comparison, hydraulic fracture in a full space, in which elastic interactions are nonlocal, reduces to singular integro-differential equations, requiring more specialized solution techniques. A peculiarity of the thin-sheet problem is that the inherent neglect of variations over distances on the order of the sheet thickness necessitates regularization of fluid flow near the fracture tip (Flitton and King 2004). This regularization may either take the form of a fluid lag behind the tip (Hewitt et al. 2015; Ball and Neufeld 2018; Wang and Detournay 2018) or a pre-existing thin-film of fluid, such that a rupture front is no longer precisely defined (Flitton and King 2004; Hosoi and Mahadevan 2004; Lister et al. 2013; Hewitt et al. 2015; Peng and Lister 2020), or may be bypassed altogether by explicit consideration of near-tip phenomena over distances comparable to the sheet thickness (Lister et al. 2019).

We now look to examine the shear-fracture counterpart to the above problems, in the context of fluid-induced slip of geologic faults, the thin-sheet limit of which is of interest in the modeling of landslides (Palmer and Rice 1973; Puzrin and Germanovich 2005; Viesca and Rice 2012), snow-slab avalanches (McClung 1979), and short-timescale icesheet motion (Lipovsky and Dunham 2017), among other problems. A source of fluid in the subsurface can drive the sliding-mode fracture of a geologic fault by locally reducing the fault's frictional shear strength below an ambient level of shear stress such that fault must slide. The fluid source may be natural, such as from mineral dehydration of subducted sediments (Peacock 2001) or from a mantle source (Kennedy et al. 1997), or artificial, such as from the subsurface injection of fluids at kilometer scale depths for the disposal of wastewater (Healy et al. 1968) or for the enhancement of permeability in geothermal, oil, or gas reservoirs. Despite wide interest, simple solutions for fluid-induced fault slip are scarce.

In the context of frictional fracture, much of the development of fluid-fracture interaction has focused on geologic fault phenomena, including earthquakes and slow, aseismic slip. Fluid-driven fault slip has been studied in the context of earthquake nucleation via the initiation of aseismic slip (Viesca and Rice 2012; Garagash and Germanovich 2012; Jha and Juanes 2014; Ciardo and Lecampion 2019; Zhu et al. 2020; Garagash 2021), or strictly stable, aseismic slip (Rutqvist et al. 2007; Garipov et al. 2016; Bhattacharya and Viesca 2019; Dublanchet 2019; Yang and Dunham 2021). In addition, there has been substantial focus on fluid-involved feedback mechanisms during seismic or aseismic fault rupture, in which the role of fluids is in response to sliding such that rupture is fluid-assisted or fluid-inhibited. These including dilatancy (Rice 1973; Yang and Dunham 2021; Brantut 2021), thermal pressurization by frictional heating (Rice 2006; Noda et al. 2009; Schmitt et al. 2011; Garagash 2012; Viesca and Garagash 2015) and chemical decomposition (Platt et al. 2015). Within this body of work, solutions are nearly entirely numerical.

We examine a model for fluid-driven fault rupture, in which the condition of fluid flow is rudimentary but physically plausible and comparable to the starting assumption of laminar flow in hydraulic fracture. We consider a planar fault in an unbounded, linear-elastic medium under a uniform state of stress prior to injection. Quasi-static deformation is in-plane or anti-plane, such that the corresponding fault slip is mode II or mode III rupture. We use a boundary integral formulation to relate the crack-face displacement and traction. Injection is modeled as a line source of fluids at constant pressure following which fluid migration, and the concomitant rise in pore fluid pressure, is restricted to occur along the fault plane. The fault strength is frictional and is the product of a constant coefficient of friction and the local 
effective normal stress, the difference between the fault-normal traction and the local pore fluid pressure.

This problem was presented in Bhattacharya and Viesca (2019) and is a variation of one considered in depth by Garagash and Germanovich (2012) (hereafter referred to as GG12). GG12 examined the response to injection of a fault under the same elastic and fluid conditions considered here. However, GG12 considered a fault friction coefficient that weakens with slip, a feature that gives rise to rich behavior, including the possibility of dynamic rupture nucleation and arrest, corresponding to an earthquake source. GG12 found two end-member regimes corresponding to marginally pressurized and critically stressed faults, which reflect the pre-injection state of stress. The authors showed that these regimes lead to a rupture front lagging or outpacing fluid diffusion, respectively, and that, in the critically stressed regime, fault slip can be described by a boundary-layer analysis. However the slip-dependent strength in that problem required numerical solution, even in end-member regimes, and growth of the slipping region was non-trivial and dependent on at least two problem parameters.

A constant Coulomb friction coefficient necessitates stable growth of fault rupture. Furthermore, the propagation of fault slip occurs in self-similar manner in response to a self-similar source. Additionally, the problem has a single dimensionless parameter, with the same two end-member regimes as in the problem considered by GG12. The simpler problem admits closed-form perturbation expansions in these regimes, and readily allows for higher-order asymptotic matching of the boundary-layer problem in the critically stressed regime. In this regime, where the rupture front races ahead of the elevated fluid pressure distribution, the problem provides for the development of a multipole expansion to consider the higher-order source effects. The problem is also amenable to accurate numerical solutions for the cases intervening the two end-member regimes and we provide tabulated solutions to high precision.

We begin in Sections 2 and 3 by providing a problem statement and summary of asymptotic solutions to leading order in the critically stressed and marginally pressurized limits. Subsequently, we return to the full problem and summarize its solution in Section 4. Finally, we revisit the end-member regimes and derive the asymptotic solutions to second order. In the marginally pressurized limit, the solution can be written as a single perturbation expansion (Section 5). In the critically stressed limit, inner and outer perturbation expansions are found and the two solutions are matched to construct a composite solution (Sections 6-8). We compare the asymptotic expansions to numerical solutions to the full problem throughout.

\section{Problem formulation}

\subsection{Fluid mechanics}

A planar fault slip surface along $y=0$ is modeled as an interface within a poroelastic layer of thickness $h$ that is itself embedded within an elastic body. The layer corresponds to a fault core that is presumed to be much more permeable than the surrounding host rock, but having comparable elastic properties. We assume that fluid flow within the layer follows Darcy's law and we examine the injection of fluid directly into the fault core as a source of constant pressure distributed across the layer thickness at $x=0$. For this poroelastic configuration, which is an in-plane version of an axisymmetric case considered by Marck et al. (2015), the pore fluid pressure distribution is uniform across the layer thickness and its distribution $p(x, t)$ along the fault coordinate $x$ satisfies a diffusion equation

$$
p_{t}=\alpha_{h y} p_{x x}
$$



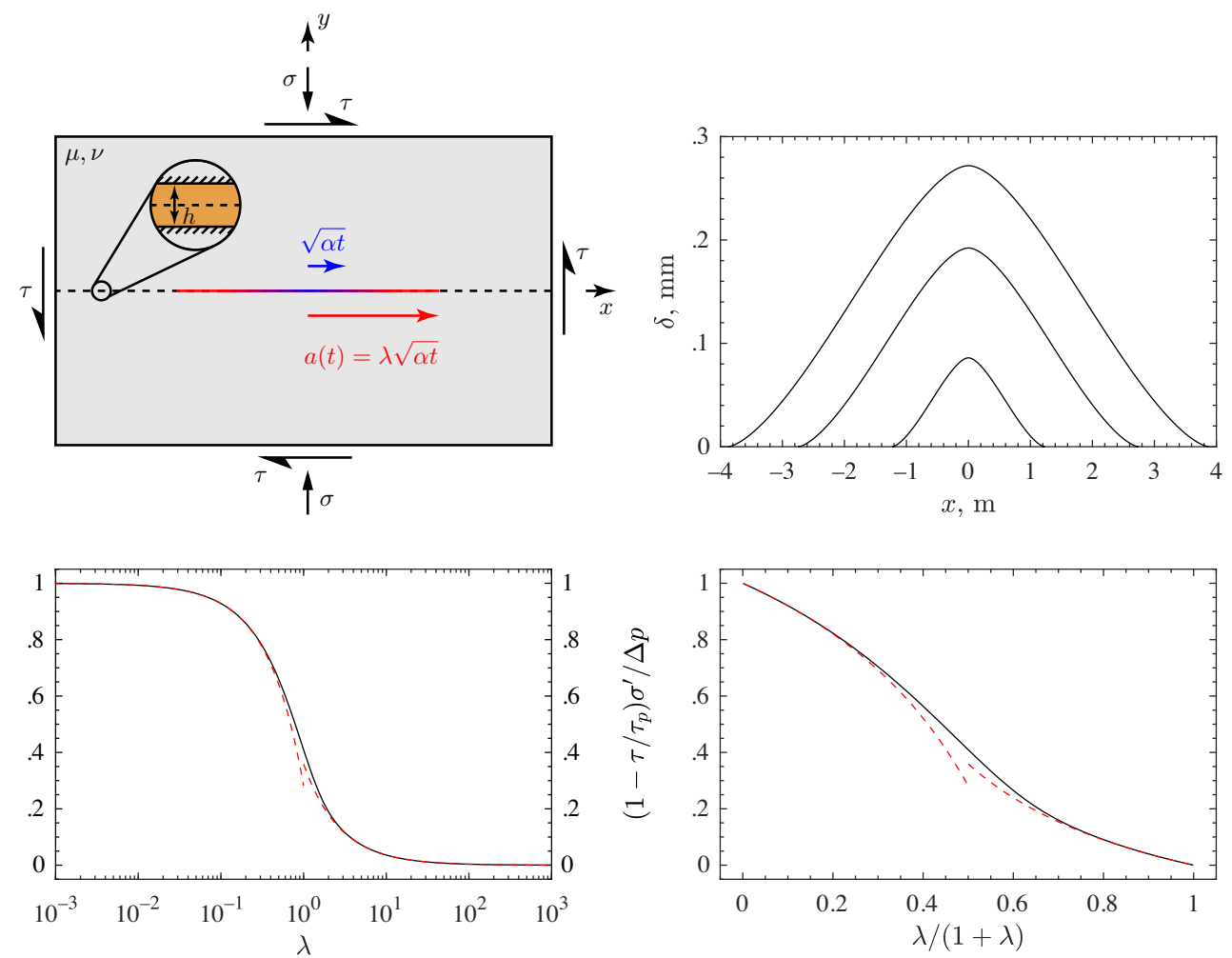

Figure 1: Counter-clockwise: (top left) Unbounded elastic body containing a fault, loaded remotely with fault-normal and shear stress $\sigma, \tau$. The fault is embedded within a thin poroelastic layer, assumed to be much more permeable than surroundings. Fluid injected at $x=0$ diffuses along fault as $\sqrt{\alpha t}$, inducing quasi-static slip out to a distance $a(t)$. Fault has constant friction coefficient $f$. (bottom left) Black: relation between rupture growth factor $\lambda$ and a parameter reflecting the initial state of stress and injection pressure, where $\sigma^{\prime}=\sigma-p_{o}$ and $p_{o}$ is pre-injection fault fluid pressure. Dashed: asymptotic behaviors, eqs. (3.2) and (3.5). (bottom right) Same as bottom left, with abscissa arranged to occupy a finite interval. (top right) Plot of self-similar slip distributions at three instants in time after the start of injection, $t=1,5$, and $10 \mathrm{~min}$., for the specific choices $\sigma=50 \mathrm{MPa}, \tau=$ $12 \mathrm{MPa}, p_{o}=20 \mathrm{MPa}, \Delta p=12 \mathrm{MPa}, f=0.5, \alpha_{h y}=0.01 \mathrm{~m}^{2} / \mathrm{s}, \mu=30 \mathrm{GPa}, v=1 / 4$, $\mu^{\prime}=20 \mathrm{GPa}$. For these choices, the parameter $\left(1-\tau / \tau_{p}\right) \sigma^{\prime} / \Delta p=0.5$. The corresponding self-similar slip distribution and factor $\lambda$ are given in Table S1 in the supplementary materials.

where $\alpha_{h y}$ is the hydraulic diffusivity of the fault core and where the pore pressure is subject to the conditions of the initial state and injection at constant pressure $\Delta p$ at $x=0$,

$$
p(x, 0)=p_{o}, \quad p(0, t>0)=\Delta p
$$

the known solution to which is

$$
p(x, t)=p_{o}+\Delta p \operatorname{erfc}(|x| / \sqrt{\alpha t})
$$

where we adopt a nominal diffusivity

$$
\alpha=4 \alpha_{\text {hy }}
$$

in which the hydraulic diffusivity $\alpha_{h y}=k /(\beta \eta)$, where $k$ is the Darcy permeability of the layer, $\eta$ is the viscosity of the permeating fluid, and $\beta$ is a storage coefficient reflecting the 
compressibility of the fluid and porous matrix. As noted by Marck et al. (2015), when the diffusive distance $\sqrt{\alpha t}$ is much larger than the layer thickness $h$, the local response of the poroelastic layer is effectively that of uniaxial vertical strain in proportion to the local pore fluid pressure.

\subsection{Solid mechanics}

We assume that the poroelastic layer thickness $h$ is small such that the condition $\sqrt{\alpha t} \gg h$ is quickly achieved. In the case, the presence of the layer, apart from its role in conducting fluids, need not be explicitly considered and the shear and normal traction conditions on the layer-medium boundary may be directly applied to the sliding interface. Furthermore, provided $\sqrt{\alpha t}$ is sufficiently greater than $h$, we may reasonably neglect any fault-normal stress changes due to the swelling of the poroelastic layer about the source (Marck et al. 2015). The medium containing the fault is assumed to be linearly elastic and its deformation, along with slip on the fault, may be in-plane or anti-plane. The in-plane case is illustrated in Fig. 1 (top left). The shear modulus of the medium is $\mu$ and the Poisson ratio $v$. We define the effective elastic modulus $\mu^{\prime}=\mu /[2(1-v)]$ for in-plane (mode-II) case and $\mu^{\prime}=\mu / 2$ for anti-plane (mode-III) case. We denote the initial (pre-injection) fault shear stress $\tau$ (in-plane or anti-plane), the fault friction coefficient $f$, the initial total fault-normal compressive stress $\sigma$, and the initial effective normal stress $\sigma^{\prime}=\sigma-p_{o}$, where $p_{o}$ is the pre-injection pore fluid pressure in the layer. The initial fault strength is $\tau_{p}=f \sigma^{\prime}$.

The fault obeys a Coulomb friction law: the local shear strength of the fault $\tau_{s}$ is a constant proportion of the local effective normal stress, with a constant coefficient of friction $f$

$$
\tau_{s}(x, t)=f[\sigma-p(x, t)]
$$

Where sliding occurs, this strength must equal the shear stress on the fault. The shear stress can be decomposed into a sum of the initial shear stress $\tau$ plus quasi-static changes due to a distribution of slip $\delta$ (e.g., Rice 1968), such that the stress-strength condition is

$$
\tau_{s}(x, t)=\tau+\frac{\mu^{\prime}}{\pi} \int_{-a(t)}^{a(t)} \frac{\partial \delta(s, t) / \partial s}{s-x} d s
$$

where $x= \pm a(t)$ are the crack-tip locations.

\section{Summary of results}

After non-dimensionalizing, the problem is found to have the sole parameter

$$
\left(1-\frac{\tau}{\tau_{p}}\right) \frac{\sigma^{\prime}}{\Delta p}
$$

that is bounded between 0 and 1 . The upper bound denotes a marginally pressurized fault, where the fluid pressure increase is just sufficient to initiate sliding: $f\left[\sigma-\left(p_{o}+\Delta p\right)\right]=\tau$. The lower bound denotes a critically stressed fault, where the initial shear stress is equal to the initial shear strength: $\tau=\tau_{p}$.

The solution consists of a self-similar distribution of slip, in which the crack front grows as

$$
a(t)=\lambda \sqrt{\alpha t}
$$

and the slip distribution can be written as

$$
\delta(x, t) \Rightarrow \delta(\bar{x})
$$



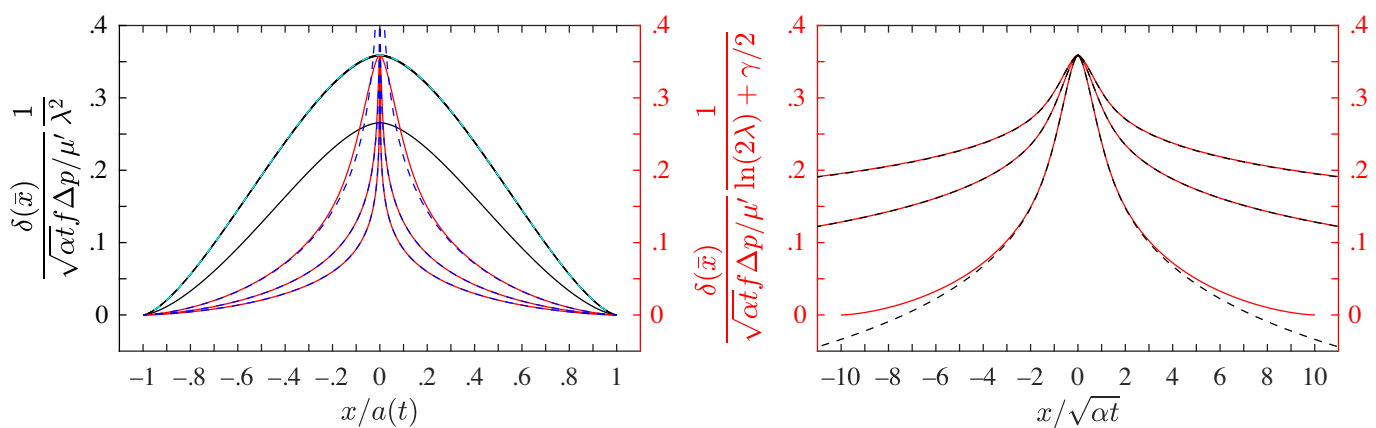

Figure 2: (left) Self-similar distributions of slip $\delta$ with distance from injection point $x$, which is scaled by the crack half-length $a(t)=\lambda \sqrt{\alpha t}$. Solid red and black curves are numerical solutions to the full problem. Dashed curves are leading-order asymptotic solutions. Each curve corresponds to one value of $\lambda$ in the range $\lambda=10^{-3}, 10^{-2}, \ldots, 10^{3}$. Black curves correspond to $\lambda=10^{-3}, 10^{-2}, 10^{-1}, 10^{0}$ from top to bottom, with the first three indistinguishable on this scale; red curves correspond to $\lambda=10^{1}, 10^{2}, 10^{3}$ from top to bottom. Cyan-dashed: solution for small $\lambda$, eq. (3.3). Blue-dashed: "outer" solutions for large $\lambda$, eq. (3.6). To facilitate comparisons, two vertical scales are used: one for black and cyan-dashed curves, and another for red and blue-dashed curves. (right) For large values of $\lambda$, the distribution of slip is plotted over distances scaled by $\sqrt{\alpha t}$, which is much smaller than the crack length $a(t)$. This "inner" behavior is described by eq. (3.7), a single numerical solution shown here as black-dashed curves. Curves correspond to $\lambda=10^{1}, 10^{2}, 10^{3}$ from bottom to top.

where the similarity coordinate is

$$
\bar{x}=x / a(t)
$$

The factor $\lambda$, to be solved for, determines whether the crack lags $(\lambda<1)$ or outpaces $(\lambda>1)$ the diffusion of pore pressure, which stretches as $\sqrt{\alpha t} . \lambda$ depends uniquely on the sole parameter (3.1), and that dependence is illustrated in Fig. 1b and tabulated at the top of Table $\mathrm{S} 1$ in the supplementary materials. The self-similar profile of slip, as it depends on $|x| / a(t)$, is also presented in the bottom of Table $\mathrm{S} 1$ for several values of the parameter (3.1). Scaled plots of the self-similar profile for various values of $\lambda$ are shown in Fig. 2 . In the limit that the parameter (3.1) approaches its end-member values, closed-form expressions for $\lambda$ and $\delta$ are available and provided below to leading order, with detailed derivations in the Sections that follow.

\subsection{Marginally pressurized faults, $\tau \rightarrow f\left(\sigma^{\prime}-\Delta p\right)$}

In this limit, the parameter $\left(1-\tau / \tau_{p}\right) \sigma^{\prime} / \Delta p \rightarrow 1$, the factor $\lambda \ll 1$ (i.e., the rupture lags the diffusion of pore fluid pressure), and the relation between the two follows the asymptotic expansion

$$
\left(1-\tau / \tau_{p}\right) \sigma^{\prime} / \Delta p \approx 1-\frac{4}{\pi^{3 / 2}} \lambda-O\left(\lambda^{3}\right)
$$

The slip distribution in this limit is

$$
\delta(\bar{x}) \approx \frac{\lambda^{2} \sqrt{\alpha t} f \Delta p}{\mu^{\prime}} \frac{2}{\pi^{3 / 2}}\left(\sqrt{1-\bar{x}^{2}}-\bar{x}^{2} \operatorname{atanh} \sqrt{1-\bar{x}^{2}}\right)+O\left(\lambda^{4}\right)
$$

and the accumulation of slip at the center is

$$
\delta(0) \approx \frac{2}{\pi^{3 / 2}} \frac{\lambda^{2} \sqrt{\alpha t} f \Delta p}{\mu^{\prime}}+O\left(\lambda^{4}\right)
$$



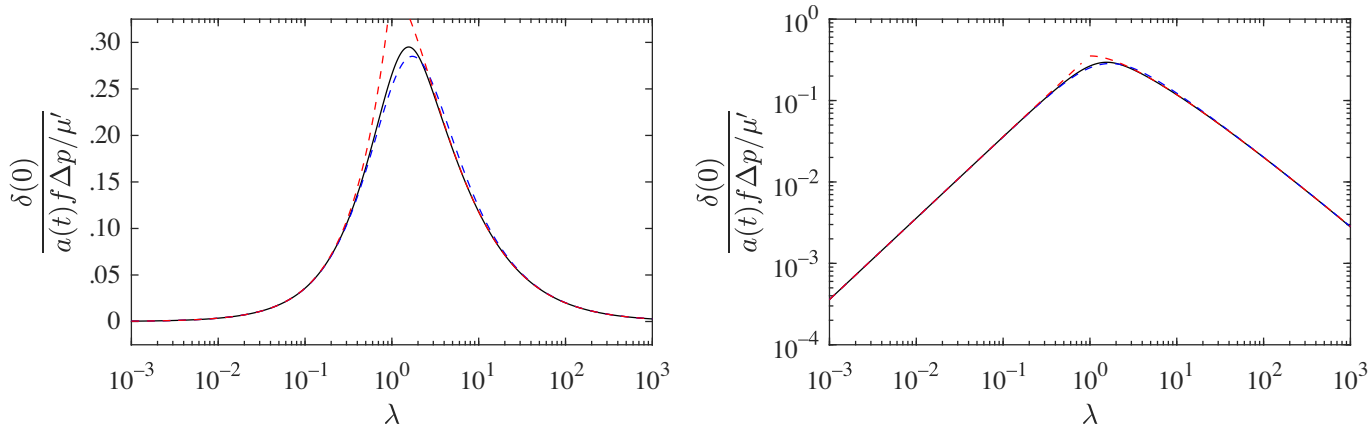

Figure 3: The maximum slip, which occurs at $x=0$, as it relates to the factor $\lambda$ shown as (left) semi-log and (right) $\log -\log$ plots. Red-dashed: end-member scalings at small $\lambda$, eq. (3.4), and at large $\lambda$, eq. (3.9). Blue-dashed: approximation of $\delta(0)$ for all $\lambda$, eq. (3.11).

The derivation of this solution is detailed in Section 5.

\subsection{Critically stressed faults, $\tau \rightarrow \tau_{p}$}

In this limit $\left(1-\tau / \tau_{p}\right) \sigma^{\prime} / \Delta p \rightarrow 0, \lambda \gg 1$ (i.e., the rupture outpaces the diffusion of fluid pressure), and the asymptotic relation is

$$
\left(1-\tau / \tau_{p}\right) \sigma^{\prime} / \Delta p \approx \frac{2}{\pi^{3 / 2}} \frac{1}{\lambda}+O\left(\lambda^{-3}\right)
$$

Similarly to the problem considered by Garagash and Germanovich (2012), the solution for slip can be decomposed into an outer solution on distances comparable to the rupture distance $a(t)$, and an inner solution on distances comparable to the diffusion lengthscale $\sqrt{\alpha t}$. The two solutions are matched at an intermediate distance.

The outer solution for the slip distribution is

$$
\delta(\bar{x}) \approx \frac{\sqrt{\alpha t} f \Delta p}{\mu^{\prime}} \frac{2}{\pi^{3 / 2}}\left(\operatorname{atanh} \sqrt{1-\bar{x}^{2}}-\sqrt{1-\bar{x}^{2}}\right)+O\left(\lambda^{-2}\right)
$$

where $\bar{x}$ is the similarity coordinate used above. The derivation of this solution may be found in Section 6.

The inner solution is given by the expression

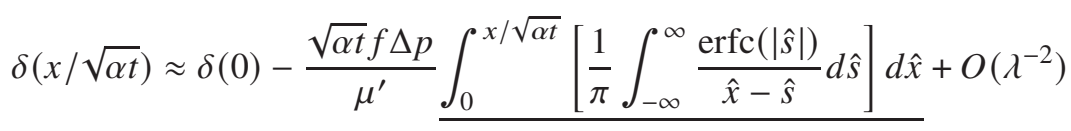

The underlined portion is evaluated numerically and provided as a supplementary function $f(x / \sqrt{\alpha t})$ in Table $\mathrm{S} 2$ with the similarity coordinate

$$
\hat{x}=\frac{x}{\sqrt{\alpha t}}
$$

For large distances $x / \sqrt{\alpha t}, f$ behaves as

$$
f(\hat{x}) \approx \frac{2}{\pi^{3 / 2}}\left(\ln |\hat{x}|+\frac{\gamma}{2}+1\right)+O\left(\hat{x}^{-2}\right)
$$

where $\gamma=0.57721566 \ldots$ is the Euler-Maraschoni constant. Using this asymptotic behavior 


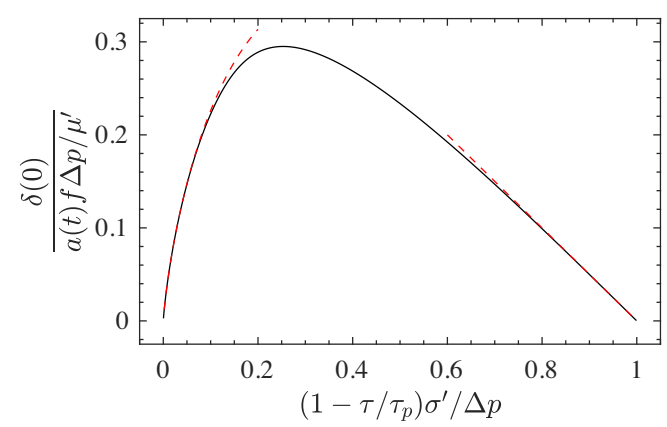

Figure 4: The peak slip as it relates to the problem parameter $\left(1-\tau / \tau_{p}\right) \sigma^{\prime} / \Delta p$. Reddashed: scalings as the parameter approaches its bounds, derived from eqs. (3.4) and (3.9) and the asymptotic relations between the parameter and $\lambda$, eqs. (3.2) and (3.5).

to match the inner solution at large $x / \sqrt{\alpha t}$ with the outer solution at small $x / a(t)$ provides the slip at the center

$$
\delta(0) \approx \frac{\sqrt{\alpha t} f \Delta p}{\mu^{\prime}} \frac{2}{\pi^{3 / 2}}\left[\ln (2 \lambda)+\gamma / 2+O\left(\lambda^{-2}\right)\right]
$$

in the large $\lambda$ limit.

Other properties of $f(x / \sqrt{\alpha t})$ include

$$
f^{\prime \prime}(\hat{x})=-\frac{2}{\pi^{3 / 2}} \exp \left(-\hat{x}^{2}\right) \operatorname{Ei}\left(\hat{x}^{2}\right)
$$

where $\operatorname{Ei}(x)=-\int_{-x}^{\infty} \exp (-u) / u d u$ is the exponential integral, and in the limit that $x / \sqrt{\alpha t}$ is small, $f$ behaves as

$$
f(\hat{x}) \approx \frac{2}{\pi^{3 / 2}} \hat{x}^{2}\left(\ln \frac{1}{|\hat{x}|}-\frac{\gamma}{2}+\frac{3}{2}\right)+O\left(\hat{x}^{4} \ln |\hat{x}|\right)
$$

A detailed discussion of the inner solution and its matching to the outer solution, can be found in Sections 7 and 8.

\subsection{Accumulation of slip at the injection point}

Figs. 3 and 4 show the solution for the peak slip, located at the injection point, as it depends on the parameter (3.1) or the factor $\lambda$. An approximation of peak slip at the injection point that respects the asymptotic behavior at both critically stressed and marginally pressurized limits—eqs. (3.4) and (3.9) —and is to within 5\% error over the intervening range of $\lambda$, is

$$
\delta(0) \approx \frac{\lambda \sqrt{\alpha t} f \Delta p}{\mu^{\prime}} \frac{2}{\pi^{3 / 2}} \frac{\lambda}{1+\lambda^{2} /[\ln (6+2 \lambda)+\gamma / 2]}
$$

\section{Non-dimensionalization and solution to full problem}

Combining eqs. (2.1)-(2.3) and rearranging leads to the non-dimensionalized equation

$$
\left(1-\frac{\tau}{\tau_{p}}\right) \frac{\sigma^{\prime}}{\Delta p}-\operatorname{erfc}|\lambda \bar{x}|=-\frac{1}{\pi} \int_{-1}^{1} \frac{d \bar{\delta} / d \bar{s}}{\bar{x}-\bar{s}} d \bar{s}
$$




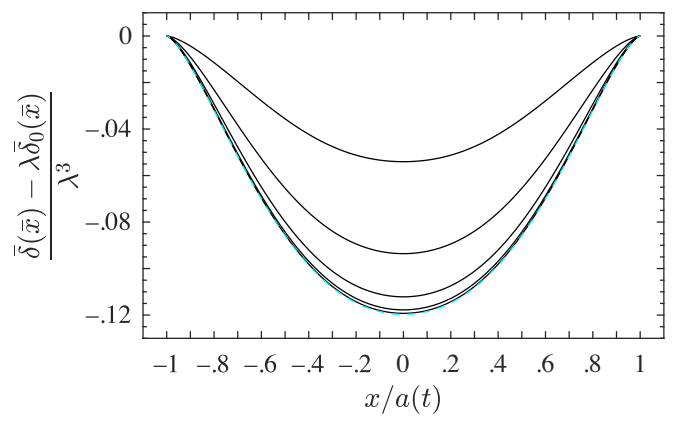

Figure 5: Self-similar distribution of slip $\delta$, less the first-order term, eq. (5.5), in the asymptotic expansion for slip eq. (5.4) in the marginally pressurized limit (small $\lambda$ ). From top to bottom, black curves correspond to the difference for $\lambda=2,1, \frac{1}{2}, \frac{1}{4}, \frac{1}{8}$. Cyan-dashed curve is the second-order term of the expansion, eq. (5.6)

where we have used $x=a(t) \bar{x}, a(t)=\lambda \sqrt{\alpha t}$, and $\delta(x, t)=\bar{\delta}[x / a(t)] a(t) f \Delta p / \mu^{\prime}$. The solution we seek is the slip distribution $\bar{\delta}$ and the crack-growth prefactor $\lambda$, including their dependence on the problem parameter $\left(1-\tau / \tau_{p}\right) / \sigma^{\prime} / \Delta p$.

We begin by looking for the solution for $\lambda$. To do so, we first note that to avoid a singularity in shear stress ahead of the crack tips, which is necessary because the Coulomb friction requirement implies a finite shear strength of the interface, the crack-tip stress intensity factors of the rupture must be zero. This condition implies (Appendix A)

$$
\left(1-\frac{\tau}{\tau_{p}}\right) \frac{\sigma^{\prime}}{\Delta p}=\frac{1}{\pi} \int_{-1}^{1} \frac{\operatorname{erfc}|\lambda x|}{\sqrt{1-x^{2}}} d x
$$

which provides an implicit solution for $\lambda$ as it depends on the problem parameter. This relation is easily determined numerically since, for a given $\lambda$, the integrand on the right hand side can be evaluated by Gauss-Chebyshev quadrature (Appendix B). The behavior at large and small values of $\lambda$, eqs. (3.2) and (3.5), is found by asymptotic approximation of the integral.

To solve for $\bar{\delta}$, we note that (4.1) may be inverted for $d \bar{\delta} / d \bar{x}$ (Appendix A)

$$
\frac{d \bar{\delta}}{d \bar{x}}=-\frac{\sqrt{1-\bar{x}^{2}}}{\pi} \int_{-1}^{1} \frac{\operatorname{erfc}|\lambda \bar{s}|}{\sqrt{1-\bar{s}^{2}}} \frac{1}{\bar{x}-\bar{s}} d \bar{s}
$$

After having determined $\lambda$ via (4.2), the right hand side may be numerically evaluated and integrated to arrive to $\bar{\delta}(\bar{x})$ using a Gauss-Chebyshev quadrature for singular integrals (Erdogan et al. 1973; Viesca and Garagash 2018). The numerical solution of (4.2) and (4.3) for $\lambda$ and $\bar{\delta}(\bar{x})$ is detailed in Appendix B.

\section{Solution in the marginally pressurized limit}

For $\lambda \ll 1$, we may use the expansion of the function

$$
\operatorname{erfc}|\lambda \bar{x}| \approx 1-\frac{2}{\sqrt{\pi}}|\lambda \bar{x}|+\frac{2}{3 \sqrt{\pi}}|\lambda \bar{x}|^{3}+\ldots
$$

to expand the integral in eq. (4.2) as

$$
\left(1-\frac{\tau}{\tau_{p}}\right) \frac{\sigma^{\prime}}{\Delta p}=\int_{-1}^{1} \frac{\operatorname{erfc}|\lambda x|}{\sqrt{1-x^{2}}} d x \approx 1-\frac{4}{\pi^{3 / 2}} \lambda+\frac{8}{9 \pi^{3 / 2}} \lambda^{3}+O\left(\lambda^{5}\right)
$$




\begin{tabular}{c|c}
$g(x)$ & $h(x)$ \\
\hline 1 & $\frac{1}{\pi} \frac{\sqrt{1-x^{2}}}{x^{2}}$ \\
$\delta_{D}^{\prime \prime}(x)$ & $-\frac{1}{\pi} \frac{\sqrt{1-x^{2}}}{x}$ \\
$\delta_{D}^{\prime}(x)$ & $\frac{1}{\pi} \operatorname{atanh} \sqrt{1-x^{2}}$ \\
$\delta_{D}(x)$ & $\frac{1}{\pi} x \operatorname{atanh} \sqrt{1-x^{2}}$ \\
$\operatorname{sign}(x) / 2$ & $\frac{1}{\pi} x^{2} \operatorname{atanh} \sqrt{1-x^{2}}$ \\
$|x|-\frac{1}{\pi}$ & $\frac{2}{3 \pi} x^{3} \operatorname{atanh} \sqrt{1-x^{2}}+\frac{2}{3 \pi} x \sqrt{1-x^{2}}$ \\
$|x| x$ & $\frac{1}{3 \pi} x^{4} \operatorname{atanh} \sqrt{1-x^{2}}+\frac{1}{2 \pi} x^{2} \sqrt{1-x^{2}}$
\end{tabular}

Table 1: Select solutions $h(x)$ to the problem $g(x)=\frac{1}{\pi} \int_{-1}^{1} \frac{h^{\prime}(s)}{x-s} d s$, with $h( \pm 1)=0$.

In turn, we use eqns. (5.1) and (5.2) to reduce eq. (4.1) to

$$
\frac{1}{\pi} \int_{-1}^{1} \frac{d \bar{\delta} / d \bar{s}}{\bar{x}-\bar{s}} d \bar{s} \approx \lambda\left(\frac{4}{\pi^{3 / 2}}-\frac{2}{\sqrt{\pi}}|\bar{x}|\right)+\lambda^{3}\left(-\frac{8}{9 \pi^{3 / 2}}+\frac{2}{3 \sqrt{\pi}}|x|^{3}\right)+O\left(\lambda^{5}\right)
$$

We write the solution to the above equation as the perturbation expansion

$$
\bar{\delta}(\bar{x}) \approx \lambda \delta_{0}(\bar{x})+\lambda^{3} \delta_{1}(\bar{x})+O\left(\lambda^{5}\right)
$$

where $\delta_{0}$ and $\delta_{1}$ satisfy

$$
\frac{1}{\pi} \int_{-1}^{1} \frac{d \delta_{0} / d \bar{s}}{\bar{x}-\bar{s}} d \bar{s}=\frac{4}{\pi^{3 / 2}}-\frac{2}{\sqrt{\pi}}|\bar{x}|, \quad \frac{1}{\pi} \int_{-1}^{1} \frac{d \delta_{1} / d \bar{s}}{\bar{x}-\bar{s}} d \bar{s}=-\frac{8}{9 \pi^{3 / 2}}-\frac{2}{3 \sqrt{\pi}}|\bar{x}|^{3}
$$

The solutions to which

$$
\begin{gathered}
\delta_{0}(\bar{x})=\frac{2}{\pi^{3 / 2}}\left(\sqrt{1-\bar{x}^{2}}-\bar{x}^{2} \operatorname{atanh} \sqrt{1-\bar{x}^{2}}\right) \\
\delta_{1}(\bar{x})=\frac{1}{3 \pi^{3 / 2}}\left(\left(\bar{x}^{2}-2\right) \sqrt{1-\bar{x}^{2}}+\bar{x}^{4} \operatorname{atanh} \sqrt{1-\bar{x}^{2}}\right)
\end{gathered}
$$

are found by the linear superposition of particular solutions to the general problem

$$
g(x)=\frac{1}{\pi} \int_{-1}^{1} \frac{h^{\prime}(s)}{x-s} d s
$$

that are provided in Table 1 and found following Appendix A. Using (5.4), the slip at the injection point in the marginally pressurized limit evaluates to

$$
\delta(0) \approx \frac{\lambda \sqrt{\alpha t} f \Delta p}{\mu^{\prime}}\left(\frac{2}{\pi^{3 / 2}} \lambda-\frac{2}{3 \pi^{3 / 2}} \lambda^{3}+O\left(\lambda^{5}\right)\right)
$$




\section{Outer solution in the critically stressed limit}

We now look for an asymptotic expansion of the solution in the critically stressed limit, in which the rupture front outpaces fluid pressure diffusion, $\lambda \gg 1$. As noted by GG12 for their problem, the solution consists of an outer solution on distances comparable to the rupture distance $a(t)$ and an inner solution on distances comparable to $\sqrt{\alpha t}$. To look for the outer solution we solve for the slip distribution satisfying eq. (4.1) after expanding the two terms on the left hand side in the large $\lambda$ limit. We begin by considering the expansion of the following function as $\lambda \rightarrow \infty$

$$
\frac{\operatorname{erfc}|u|}{\sqrt{1-(u / \lambda)^{2}}} \approx \operatorname{erfc}|u|+\frac{1}{2 \lambda^{2}} u^{2} \operatorname{erfc}|u|+O\left(\lambda^{-4}\right)
$$

This function appears in the integral (4.2) following the change of variable $u=\lambda \bar{x}$, such that

$$
\left(1-\frac{\tau}{\tau_{p}}\right) \frac{\sigma^{\prime}}{\Delta p}=\frac{1}{\pi \lambda} \int_{-\lambda}^{\lambda} \frac{\operatorname{erfc}|u|}{\sqrt{1-(u / \lambda)^{2}}} d u \approx \frac{2}{\pi^{3 / 2}} \frac{1}{\lambda}+\frac{1}{3 \pi^{3 / 2}} \frac{1}{\lambda^{3}}+O\left(\lambda^{-5}\right)
$$

In addition, we perform a multipole expansion of the distribution (Appendix C)

$$
\operatorname{erfc}|\lambda \bar{x}| \approx p_{0} \delta_{D}(\bar{x})-p_{1} \delta_{D}^{\prime}(\bar{x})+p_{2} \delta_{D}^{\prime \prime}(\bar{x})+O\left(\lambda^{-5}\right)
$$

where $\delta_{D}$ is the Dirac delta and its first and second derivatives, $\delta_{D}^{\prime}(x)$ and $\delta_{D}^{\prime \prime}(x)$, with the properties

$$
\int_{-\infty}^{\infty} \delta_{D}(x) d x=1, \quad \int_{-\infty}^{\infty} x \delta_{D}^{\prime}(x) d x=1, \quad \int_{-\infty}^{\infty} \frac{x^{2}}{2} \delta_{D}^{\prime \prime}(x) d x=1
$$

and where the coefficients

$$
\begin{aligned}
& p_{0}=\int_{-1}^{1} \operatorname{erfc}|\lambda \bar{x}| d \bar{x}=\frac{1}{\lambda} \int_{-\lambda}^{\lambda} \operatorname{erfc}|\hat{x}| d \hat{x}=\frac{2}{\sqrt{\pi}} \frac{1}{\lambda}+O\left(\exp \left(-\lambda^{2}\right) / \lambda\right) \\
& p_{1}=\int_{-1}^{1} \bar{x} \operatorname{erfc}|\lambda \bar{x}| d \bar{x}=0 \\
& p_{2}=\int_{-1}^{1} \frac{\bar{x}^{2}}{2} \operatorname{erfc}|\lambda \bar{x}| d \bar{x}=\frac{1}{3 \sqrt{\pi}} \frac{1}{\lambda^{3}}+O\left(\exp \left(-\lambda^{2}\right) / \lambda^{3}\right)
\end{aligned}
$$

exhibit beyond-all-orders decay at large $\lambda$ at the rate $\exp \left(-\lambda^{2}\right)$, following the leading-order term. With eqs. (6.2) and (6.3), the equation governing the slip distrbution (4.1) becomes

$$
\frac{1}{\pi} \int_{-1}^{1} \frac{d \bar{\delta} / d \bar{s}}{\bar{x}-\bar{s}} d \bar{s} \approx \frac{1}{\lambda}\left(-\frac{2}{\pi^{3 / 2}}+\frac{2}{\sqrt{\pi}} \delta_{D}(\bar{x})\right)+\frac{1}{\lambda^{3}}\left(-\frac{1}{3 \pi^{3 / 2}}+\frac{1}{3 \sqrt{\pi}} \delta_{D}^{\prime \prime}(\bar{x})\right)+O\left(\lambda^{-5}\right)
$$

As for the marginally pressurized case, we look for a solution in the form of a perturbation expansion

$$
\bar{\delta}(\bar{x}) \approx \frac{1}{\lambda} \delta_{0}(\bar{x})+\frac{1}{\lambda^{3}} \delta_{1}(\bar{x})+O\left(\lambda^{-5}\right)
$$

where $\delta_{0}$ and $\delta_{1}$ now satisfy

$$
\frac{1}{\pi} \int_{-1}^{1} \frac{d \delta_{0} / d \bar{s}}{\bar{x}-\bar{s}} d \bar{s}=-\frac{2}{\pi^{3 / 2}}+\frac{2}{\sqrt{\pi}} \delta_{D}(\bar{x}), \quad \frac{1}{\pi} \int_{-1}^{1} \frac{d \delta_{1} / d \bar{s}}{\bar{x}-\bar{s}} d \bar{s}=-\frac{1}{3 \pi^{3 / 2}}+\frac{1}{3 \sqrt{\pi}} \delta_{D}^{\prime \prime}(\bar{x})
$$

and the solutions

$$
\delta_{0}(\bar{x})=\frac{2}{\pi^{3 / 2}}\left(\operatorname{atanh} \sqrt{1-\bar{x}^{2}}-\sqrt{1-\bar{x}^{2}}\right)
$$


12

$$
\delta_{1}(\bar{x})=\frac{1}{3 \pi^{3 / 2}}\left(\frac{\sqrt{1-\bar{x}^{2}}}{\bar{x}^{2}}-\sqrt{1-\bar{x}^{2}}\right)
$$

are again found by superposing solutions provided in Table 1.

From the above, the outer solution for slip near $\bar{x}=0$ has the behavior

$$
\delta(\bar{x}) \approx \frac{\lambda \sqrt{\alpha t} f \Delta p}{\mu^{\prime}}\left[\frac{1}{\lambda} \frac{2}{\pi^{3 / 2}}\left(\log \frac{2}{|\bar{x}|}-1+\frac{\bar{x}^{2}}{4}+O\left(\bar{x}^{4}\right)\right)+\frac{1}{\lambda^{3}} \frac{1}{3 \pi^{3 / 2}}\left(\frac{1}{\bar{x}^{2}}-\frac{3}{2}+O\left(\bar{x}^{2}\right)\right)+O\left(\lambda^{-5}\right)\right]
$$

\section{Inner solution in the critically stressed limit}

To examine the behavior of slip on the diffusive lengthscale $\sqrt{\alpha t}$, we perform a change of variable to the scale distance $\hat{x}=\lambda \bar{x}=x / \sqrt{\alpha t}$, such that eq. (4.3) becomes

$$
\lambda \frac{d \bar{\delta}}{d \hat{x}}=-\frac{\sqrt{1-(\hat{x} / \lambda)^{2}}}{\pi} \int_{-\lambda}^{\lambda} \frac{\operatorname{erfc}|\hat{s}|}{\sqrt{1-(\hat{s} / \lambda)^{2}}} \frac{1}{\hat{x}-\hat{s}} d \hat{s}
$$

Rescaling slip as $\hat{\delta}=\lambda \bar{\delta}=\delta /\left(\sqrt{\alpha t} f \Delta p / \mu^{\prime}\right)$, we perform a series expansion of the square-root terms for large $\lambda$

$$
\frac{d \hat{\delta}}{d \hat{x}} \approx-\frac{1}{\pi}\left(1-\frac{1}{\lambda^{2}} \frac{\hat{x}^{2}}{2}+O\left(\lambda^{-4}\right)\right) \int_{-\lambda}^{\lambda}\left(\operatorname{erfc}|\hat{s}|+\frac{1}{\lambda^{2}} \frac{\hat{s}^{2}}{2} \operatorname{erfc}|\hat{s}|+O\left(\lambda^{-4}\right)\right) \frac{1}{\hat{x}-\hat{s}} d \hat{s}
$$

and regroup terms of similar order

$$
\frac{d \hat{\delta}}{d \hat{x}} \approx-\frac{1}{\pi} \int_{-\lambda}^{\lambda} \frac{\operatorname{erfc}|\hat{s}|}{\hat{x}-\hat{s}} d \hat{s}+\frac{1}{\lambda^{2}}\left(\frac{\hat{x}^{2}}{2} \frac{1}{\pi} \int_{-\lambda}^{\lambda} \frac{\operatorname{erfc}|\hat{s}|}{\hat{x}-\hat{s}} d \hat{s}-\frac{1}{\pi} \int_{-\lambda}^{\lambda} \frac{\left(\hat{s}^{2} / 2\right) \operatorname{erfc}|\hat{s}|}{\hat{x}-\hat{s}} d \hat{s}\right)+O\left(\lambda^{-4}\right)
$$

Given the beyond-all-orders decay, for modestly large values $\hat{s}$, of the term erfc $|\hat{s}| \approx$ $\exp \left(-\hat{s}^{2}\right) /(\sqrt{\pi} \hat{s})$ appearing in all of the integrands above, the limits of the integrals may pass to $\infty$ without consequence for the above expansion in powers of $\lambda$. Hence,

$$
\frac{d \hat{\delta}}{d \hat{x}} \approx-\frac{1}{\pi} \int_{-\infty}^{\infty} \frac{\operatorname{erfc}|\hat{s}|}{\hat{x}-\hat{s}} d \hat{s}+\frac{1}{\lambda^{2}}\left(\frac{\hat{x}^{2}}{2} \frac{1}{\pi} \int_{-\infty}^{\infty} \frac{\operatorname{erfc}|\hat{s}|}{\hat{x}-\hat{s}} d \hat{s}-\frac{1}{\pi} \int_{-\infty}^{\infty} \frac{\left(\hat{s}^{2} / 2\right) \operatorname{erfc}|\hat{s}|}{\hat{x}-\hat{s}} d \hat{s}\right)+O\left(\lambda^{-4}\right)
$$

The above integrals are Hilbert transforms, defined as

$$
\mathcal{H}[f(s)]=\frac{1}{\pi} \int_{-\infty}^{\infty} \frac{f(s)}{x-s} d s
$$

which have the property

$$
\begin{aligned}
\mathcal{H}\left[s^{2} f(s)\right] & =\mathcal{H}\left[\left(s^{2}-x^{2}+x^{2}\right) f(s)\right] \\
& =x^{2} \mathcal{H}[f(s)]+\mathcal{H}[(s-x)(s+x) f(s)] \\
& =x^{2} \mathcal{H}[f(s)]-\frac{1}{\pi} \int_{-\infty}^{\infty} s f(s) d s-\frac{x}{\pi} \int_{-\infty}^{\infty} f(s) d s
\end{aligned}
$$

that, when applied to the third integral in eq. (7.4)

$$
\frac{1}{\pi} \int_{-\infty}^{\infty} \frac{\left(\hat{s}^{2} / 2\right) \operatorname{erfc}|\hat{s}|}{\hat{x}-\hat{s}} d \hat{s}=\frac{\hat{x}^{2}}{2} \frac{1}{\pi} \int_{-\infty}^{\infty} \frac{\operatorname{erfc}|\hat{s}|}{\hat{x}-\hat{s}} d \hat{s}-\frac{\hat{x}}{\pi^{3 / 2}}
$$



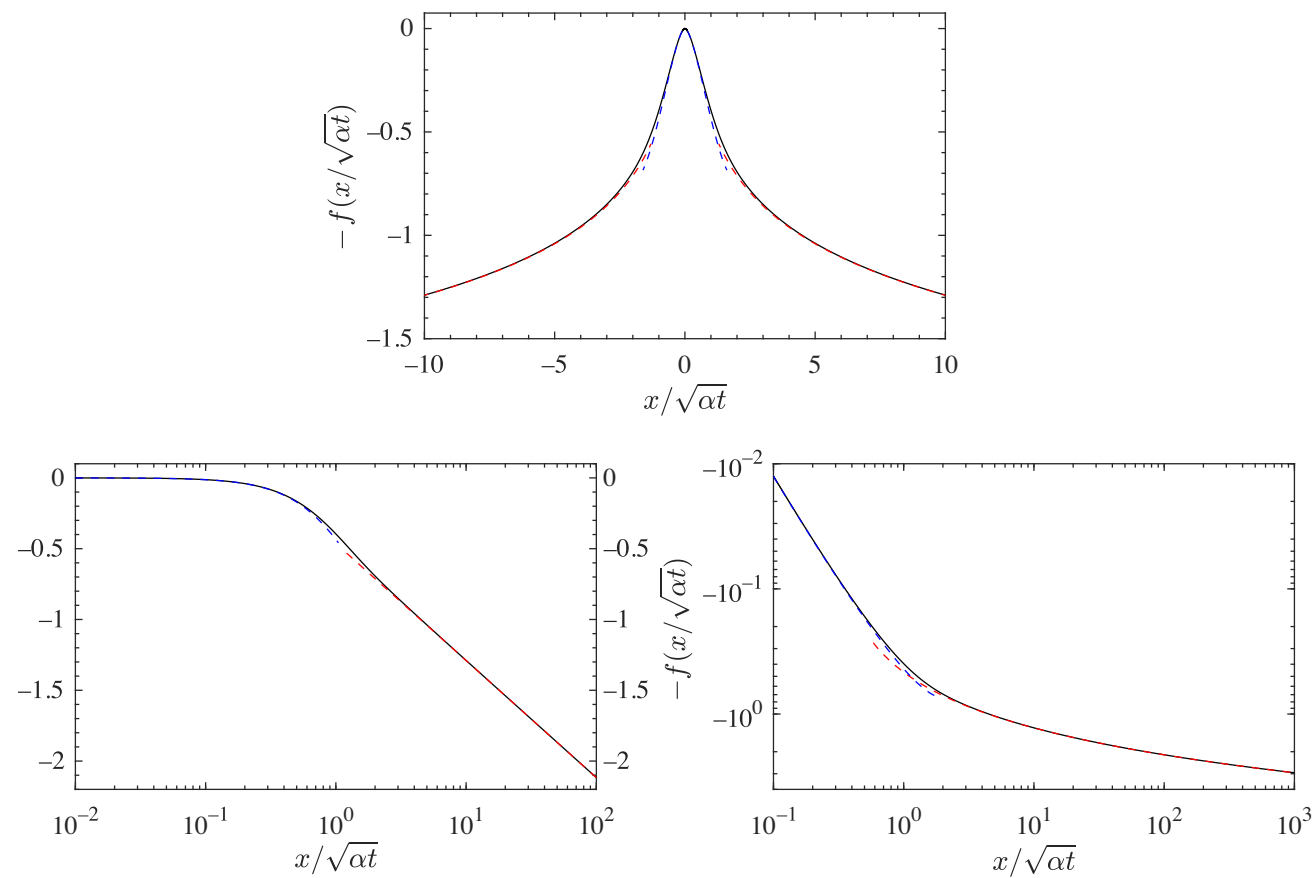

Figure 6: Spatio-temporal component, $f(x / \sqrt{\alpha t})$, of "inner" solution for slip $\delta$ in the critically stressed limit, eq. (3.7). Shown in black on linear (top), log-linear (left), and logarithmic (right) axes. Red- and blue-dashed curves: outer and inner asymptotic behavior of $f$, eqs (3.8) and (3.10).

leads to the reduction of eq. (7.4) to

$$
\frac{d \hat{\delta}}{d \hat{x}} \approx-\frac{1}{\pi} \int_{-\infty}^{\infty} \frac{\operatorname{erfc}|\hat{s}|}{\hat{x}-\hat{s}} d \hat{s}+\frac{1}{\lambda^{2}} \frac{\hat{x}}{\pi^{3 / 2}}+O\left(\lambda^{-4}\right)
$$

Subsequently integrating from 0 to $\hat{x}$, we find the inner solution to within a yet-undetermined constant $\delta(0)$

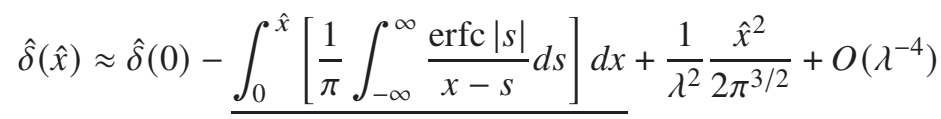

We define the underlined term as the function $f(\hat{x})$ whose first derivative is the Hilbert transform

$$
f^{\prime}(\hat{x})=\frac{1}{\pi} \int_{-\infty}^{\infty} \frac{\operatorname{erfc}|s|}{\hat{x}-s} d s
$$

Since the transform commutes with derivatives, $[\mathcal{H}(g)]^{\prime}=\mathcal{H}\left(g^{\prime}\right)$, we find that $f^{\prime \prime}$ has the concise expression

$$
f^{\prime \prime}(\hat{x})=-\frac{2}{\pi^{3 / 2}} \int_{-\infty}^{\infty} \frac{\operatorname{sign}(s) \exp \left(-s^{2}\right)}{\hat{x}-s} d s
$$



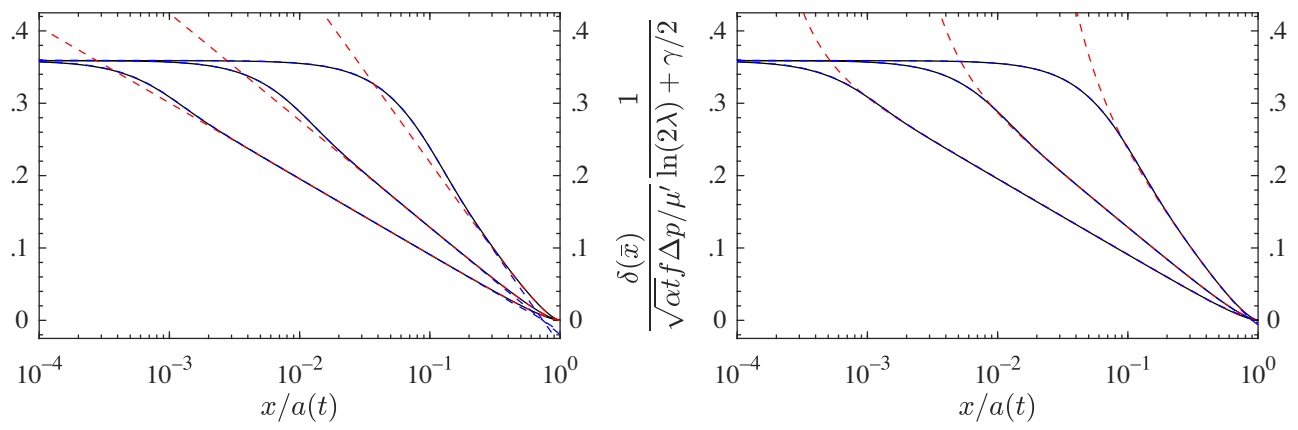

Figure 7: Self-similar distribution of slip in the critically stressed limit (large $\lambda$ ). In this limit, the rupture extent $a(t)$ outpaces the diffusive distance $\sqrt{\alpha t}$. (left and right) Black curves from right to left correspond to full solutions self-similar slip profiles for $\lambda=10,100,1000$. (left) Red-dashed and blue-dashed correspond to outer and inner solution expansion to first order. (right) Red-dashed and blue-dashed correspond to outer and inner solution expansion to second order. Outer solution is given by eqs. (6.8-6.10). Inner solution given by eq. (7.7).

which may be further simplified

$$
\begin{aligned}
f^{\prime \prime}(\hat{x}) & =-\frac{2}{\pi^{3 / 2}} \exp \left(-\hat{x}^{2}\right) \int_{0}^{\infty} \frac{\exp \left(\hat{x}^{2}-s^{2}\right)}{\hat{x}^{2}-s^{2}} 2 s d s \\
& =-\frac{2}{\pi^{3 / 2}} \exp \left(-\hat{x}^{2}\right) \int_{-\hat{x}^{2}}^{\infty} \frac{\exp (-u)}{u} d u \\
& =-\frac{2}{\pi^{3 / 2}} \exp \left(-\hat{x}^{2}\right) \operatorname{Ei}\left(\tilde{x}^{2}\right)
\end{aligned}
$$

with the change of variable $u=s^{2}-\hat{x}^{2}$ used in the intermediate step. In Fig. 6 we plot $f(\hat{x})$ found by numerically integrating twice the expression for $f^{\prime \prime}(\hat{x})$, eq. (7.8).

For $\hat{x}$ near 0 ,

$$
f^{\prime \prime}(\hat{x})=-\frac{2}{\pi^{3 / 2}}(\ln |\hat{x}|+\gamma)+O\left(\hat{x}^{2}\right)
$$

and upon integrating twice with $f(0)=f^{\prime}(0)=0$, the behavior of $f$ in this region is

$$
f(\hat{x})=\frac{2}{\pi^{3 / 2}} \hat{x}^{2}\left(\ln \frac{1}{|\hat{x}|}-\frac{\gamma}{2}+\frac{3}{2}\right)+O\left(\hat{x}^{4} \ln |\hat{x}|\right)
$$

For large $\hat{x}$

$$
f^{\prime \prime}(\hat{x})=-\frac{2}{\pi^{3 / 2}}\left(\frac{1}{\hat{x}^{2}}+\frac{1}{\hat{x}^{4}}\right)+O\left(\hat{x}^{-6}\right)
$$

Upon integrating twice with the condition that $f^{\prime}(\infty)=0$,

$$
f(\hat{x})=c+\frac{2}{\pi^{3 / 2}}\left(\ln |\hat{x}|-\frac{1}{6 \hat{x}^{2}}\right)+O\left(\hat{x}^{-4}\right)
$$

where $c$ is a yet-undetermined constant of integration.

We determine the constant $c$ following an approach used by Garagash and Germanovich (2012), in which the order of integration in eq. (7.7) is swapped leading to an alternative expression for $f(\hat{x})$

$$
f(\hat{x})=\int_{0}^{\hat{x}}\left[\frac{1}{\pi} \int_{-\infty}^{\infty} \frac{\operatorname{erfc}|s|}{x-s} d s\right] d x=\frac{1}{\pi} \int_{-\infty}^{\infty} \operatorname{erfc}|s| \ln \left|1-\frac{\hat{x}}{s}\right| d s
$$




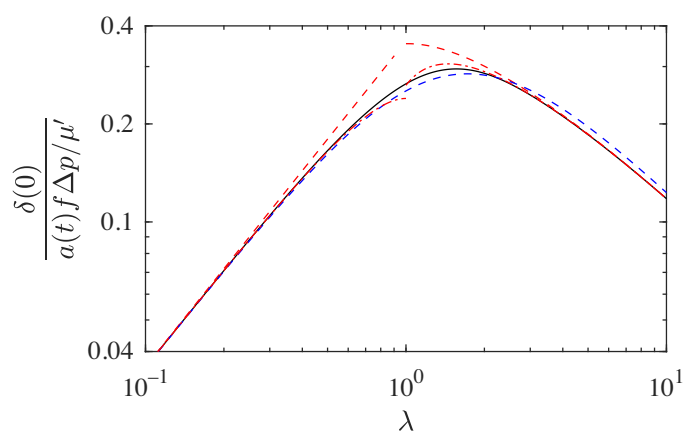

Figure 8: Comparison of approximations to the full solution of peak fault slip (black curve) in the vicinity of $\lambda=1$. First- and second- order approximations in the marginally pressurized and critically stressed limits, given respectively by eqs. (5.7) and (8.1), are shown as red-dashed and red-dot-dashed curves. Blue-dashed curve: approximation provided for all values of $\lambda$, eq. (3.11).

and the expansion $\ln |1-\hat{x} / s|=\ln |\hat{x}|-\ln |s|+\ln |s / \hat{x}-1|$ is used to decompose the latter integral for $f(\hat{x})$ into the sum

$$
\begin{aligned}
f(\hat{x}) & =\frac{1}{\pi} \int_{-\infty}^{\infty} \operatorname{erfc}|s| d s \ln |\hat{x}|-\frac{1}{\pi} \int_{-\infty}^{\infty} \operatorname{erfc}|s| \ln |s| d s+\frac{1}{\pi} \int_{-\infty}^{\infty} \operatorname{erfc}|s| \ln \left|\frac{s}{\hat{x}}-1\right| d s \\
& =\frac{2}{\pi^{3 / 2}} \ln |\hat{x}|+\frac{2}{\pi^{3 / 2}}\left(1+\frac{\gamma}{2}\right)+\frac{1}{\pi} \int_{-\infty}^{\infty} \operatorname{erfc}|s| \ln \left|\frac{s}{\hat{x}}-1\right| d s
\end{aligned}
$$

Comparing (7.9) and (7.12), we see that the asymptotic behavior of the last integral in (7.12), for large $\hat{x}$, is given by the terms in (7.9), excluding the constant and the logarithmic terms. We also retrieve the value of the constant $c$

$$
c=\frac{2}{\pi^{3 / 2}}\left(1+\frac{\gamma}{2}\right)
$$

from which we conclude that the inner solution for slip has the asymptotic behavior at large $\hat{x}$

$$
\delta(\hat{x}) \approx \delta(0)-\frac{\sqrt{\alpha t} f \Delta p}{\mu^{\prime}}\left[\frac{2}{\pi^{3 / 2}}\left(1+\frac{\gamma}{2}+\ln |\hat{x}|-\frac{1}{6|\hat{x}|^{2}}+O\left(\hat{x}^{-4}\right)\right)-\frac{1}{\lambda^{2}} \frac{\hat{x}^{2}}{2 \pi^{3 / 2}}+O\left(\lambda^{-4}\right)\right]
$$

\section{Matching inner and outer solutions}

We match the outer and inner solutions by equating eqs. (6.11) and (7.13) and solving for $\delta(0)$, the slip at the center in the critically stressed limit

$$
\delta(0) \approx \frac{\sqrt{\alpha t} f \Delta p}{\mu^{\prime}} \frac{2}{\pi^{3 / 2}}\left[\frac{\gamma}{2}+\ln (2 \lambda)-\frac{1}{4 \lambda^{2}}+O\left(\lambda^{-4}\right)\right]
$$

which completes the expression for the inner solution (7.7). In Fig. 7, we overlay the inner and outer solutions, to first and second order, above the full solutions for several large values of $\lambda$. The intermediate matching of the solutions is evident in the overlap of the dashed curves. As an aside, we can now show approximations to the slip at the center to first and second order 
in both the critically stressed and marginally pressurized limits in Fig. 8. For comparison, we also show the full solution, as well as the ad hoc approximation (3.11) constructed using the first-order asymptotics.

Using the inner and outer solutions, we construct a composite approximation (e.g., Hinch 1991)

$$
\delta_{\text {comp }}(\bar{x})=\delta_{\text {in }}(\lambda \bar{x})+\delta_{\text {out }}(\bar{x})-\delta_{\text {overlap }}(\bar{x})
$$

where $\delta_{\text {in }}$ and $\delta_{\text {out }}$ are the inner and outer solutions, eqs. (7.7) and (6.8), and

$$
\delta_{\text {overlap }}(\bar{x})=\frac{2}{\pi^{3 / 2}}\left(\log \frac{2}{\bar{x}}-1+\frac{\bar{x}^{2}}{4}\right)+\frac{1}{\lambda^{2}} \frac{1}{3 \pi^{3 / 2}}\left(\frac{1}{\bar{x}^{2}}-\frac{3}{2}\right)+O\left(\lambda^{-4}\right)
$$

is their common intermediate form. In Fig. 9 we compare the full numerical solution against the inner, outer, and composite solutions for a modestly large value of $\lambda=5$. The composite solution has an approximate error of $\mathrm{O}\left(\lambda^{-(n+2)}\right)$ where $n=2$ or 4 is the order neglected in the asymptotic expansion.

\section{Summary and conclusion}

The quasi-static rupture of a fault driven by a source of fluids has been studied in detail. Tracking the rupture of the fault corresponds to a free boundary problem for which both the size of the slipping domain and the distribution of slip within must be solved. Both depend on a single dimensionless parameter whose limits correspond either to a fault whose initial, pre-injection shear stress is relatively close to the fault's pre-injection shear strength or to a fluid pressure increase that is marginally sufficient to induce sliding. Because the problem involves contact between elastic half-spaces, interactions between points on the fault are non-local, in that slip in one location induces changes in the shear stress over the entire fault plane. The resulting equation governing slip evolution is an integro-differential equation. In addition to the crack-tip boundary condition that slip vanish at the rupture front, the condition determining the free boundary is the absence of a stress singularity ahead of the rupture front, which corresponds to the boundary condition that the gradient of slip also vanishes at the rupture front. Moreover, because the friction coefficient is held constant, there is an absence of an elasto-frictional lengthscale that may be otherwise present in problems for which friction depend explicitly on slip or its history. Correspondingly, the only lengthscale in the problem is the diffusive length $\sqrt{\alpha t}$ with the consequence that spatial dependence of slip scales directly with this lengthscale, implying the self-similar propagation of slip.

In a fashion similar to the earthquake-nucleation problem considered by GG12, we present asymptotic perturbation expansion solutions in the marginally pressurized and critically stressed limits and tabulate numerical solution for the intervening cases. In the critically stressed limit, the problem has both an inner solution on the scale of diffusion and an outer solution on the scale of the crack front. An advantage of the posed problem is that the marginally pressurized expansion and the outer solution of the critically stressed limit are expressible in concise closed form. For the latter, we develop a multipole expansion method to develop successive approximations of a distributed loading beyond a point-force approximation. The leading-order term of the inner solution of the critically stressed limit is solved numerically and tabulated. Key findings include that the rupture run-out distance from the point of injection follows $a(t)=\lambda \sqrt{\alpha t}$, where $\lambda$ is an amplification factor, originally solved for and presented by Bhattacharya and Viesca (2019). As noted there, if we denote the dimensionless problem parameter as $T \equiv\left(1-\tau / \tau_{p}\right) \sigma^{\prime} / \Delta p$, then in the marginally pressurized limit, $\lambda \approx\left(\pi^{3 / 2} / 4\right)(1-T)$ and in the critically stressed limit, $\lambda \approx\left(2 / \pi^{3 / 2}\right) T^{-1}$. 

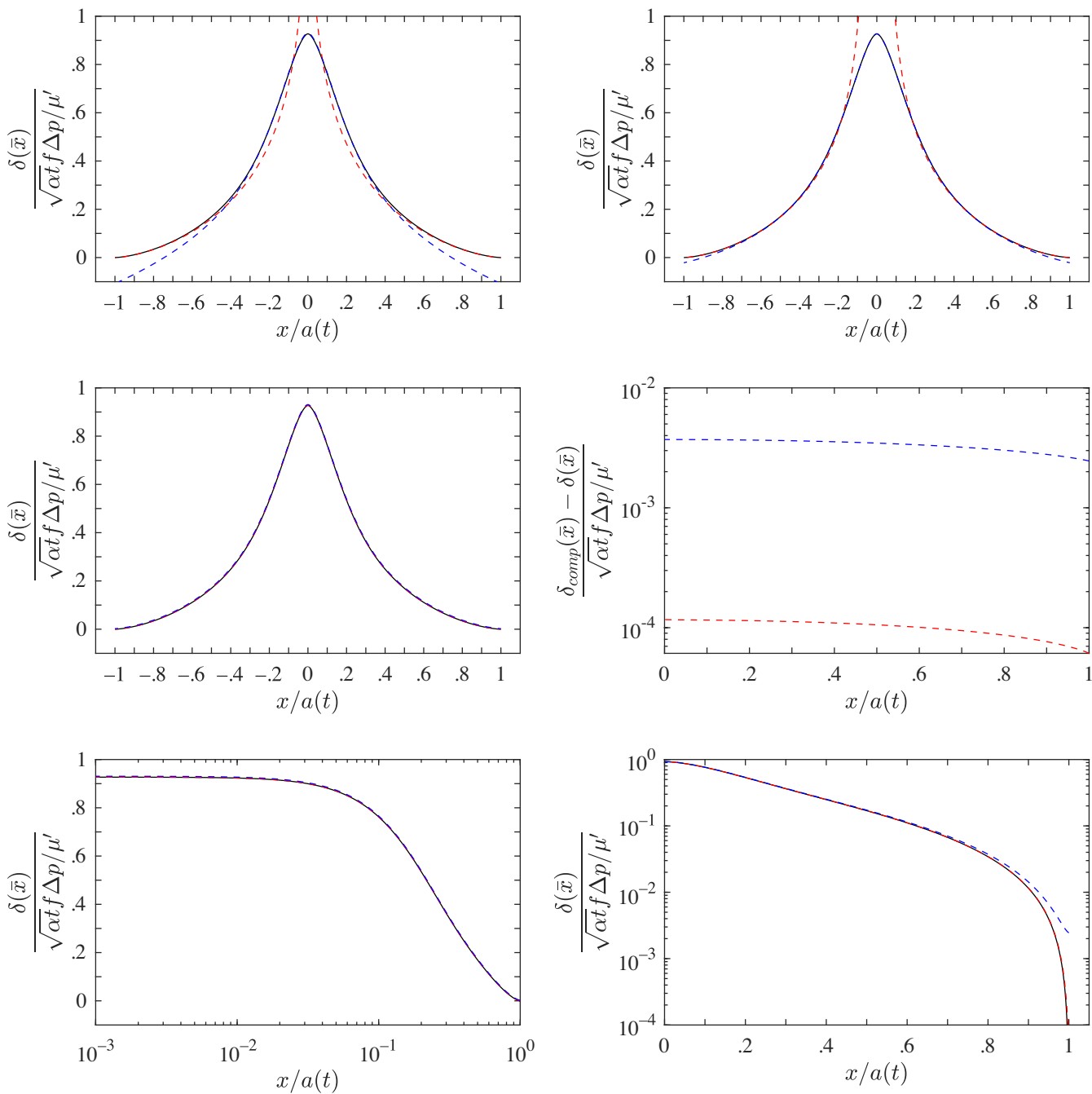

Figure 9: Solution for self-similar slip profile in black for $\lambda=5$. (top) superimposed blue- and red-dashed curves, respectively, showing inner and outer solutions to (top-left) leading and (top-order) next order. (middle-left) Superposition of leading and next-order composite solutions as blue- and red-dashed curves (middle-right). The difference between the self-similar solution and the composite solutions at leading- and next-order, respectively blue and red-dashed curves. (bottom) Semi-logarithmic plots comparing the leading- and next-order composite solutions, in blue- and red-dashed, to the self-similar solution, in black.

Furthermore, in the marginally pressurized limit, slip accumulates as $\delta \sim \lambda^{2} \sqrt{\alpha t} f \Delta p / \mu^{\prime}$. In the critically stressed limit, slip accumulates as $\delta \sim \sqrt{\alpha t} f \Delta p / \mu^{\prime}$ on inner distances of the order $\sqrt{\alpha t}$ from the injection point and as $\delta \sim \lambda^{-2} \sqrt{\alpha t} f \Delta p / \mu^{\prime}$ at distances comparable to the rupture length $\lambda \sqrt{\alpha t}$.

Supplementary data. Supplementary material is available at https://doi.org/10.1017/jfm.2019...

Funding. This work was supported by the National Science Foundation (grant number 1653382).

Declaration of interests. The authors report no conflict of interest. 
Author ORCID. R. C. Viesca, https://orcid.org/0000-0003-4180-7807

\section{Appendix A. Finite Hilbert transform solutions}

The problem for slip posed as eq. (4.1) has the form of a finite Hilbert transform

$$
g(x)=\frac{1}{\pi} \int_{-1}^{1} \frac{h^{\prime}(s)}{x-s} d s
$$

where, here, $g(x)$ corresponds to a prescribed loading and $h$ of the distribution slip on the interface that is in quasi-static equilibrium with the loading. The solution to eq. (A 1) is the known inversion (e.g., Mushkhelishvili 1958; King 2009)

$$
h^{\prime}(x)=\frac{C}{\sqrt{1-x^{2}}}-\frac{1}{\sqrt{1-x^{2}}} \frac{1}{\pi} \int_{-1}^{1} \frac{\sqrt{1-s^{2}} g(s)}{x-s} d s \quad C=\frac{1}{\pi} \int_{-1}^{1} h^{\prime}(s) d s
$$

Since slip vanishes at the rupture boundaries, the corresponding condition on $h$ is $h( \pm 1)=0$ and hence $C=0$.

As an example solution, consider the distribution $g(x)=\delta_{D}(x)$, which is equivalent to a distribution of a point-force at the origin in the corresponding crack problem. The inversion for $h^{\prime}(x)$ is

$$
h^{\prime}(x)=-\frac{1}{\sqrt{1-x^{2}}} \frac{1}{\pi} \int_{-1}^{1} \frac{\sqrt{1-s^{2}} \delta_{D}(s)}{x-s} d s=-\frac{1}{\pi} \frac{1}{x \sqrt{1-x^{2}}}
$$

and integrating again with respect to $x$, with the condition $h( \pm 1)=0$, we find that

$$
h(x)=\frac{1}{\pi} \operatorname{arctanh} \sqrt{1-x^{2}}
$$

In Table 1, we present a number of similarly derived solutions from which we draw in the main text.

From eq. (A 2), we find that $h^{\prime}(x)$ has the behavior in the limit $x \rightarrow \pm 1$

$$
h^{\prime}(x)=-\frac{1}{\sqrt{2(1 \mp x)}} \frac{1}{\pi} \int_{-1}^{1} \sqrt{\frac{1 \pm s}{1 \mp s}} g(s) d s
$$

We may compare this behavior to the leading-order term in the Williams (1957) solution for slip near the tip of a crack located at $x= \pm a$

$$
\delta(x)=\frac{K}{\mu^{\prime}} \sqrt{\frac{2(a \mp x)}{\pi}} \quad \frac{d \delta}{d x}=\mp \frac{K}{\mu^{\prime}} \sqrt{\frac{1}{2(a \mp x)}}
$$

where $K$ is the conventionally defined mode-II or mode-III stress intensity factor and the corresponding leading order term in the distribution of stress ahead of the tip is $\tau_{t i p}(x)=$ $K / \sqrt{2 \pi(x \mp a)}$. We may define a quantity $k$ corresponding to the stress intensity factor $K$ by $k=K /\left(\mu^{\prime} \sqrt{a}\right.$ ), and in comparing the latter expression in (A 6) with eq. (A 5), we can derive an analogous expression for an intensity factor $k_{ \pm}$at $x= \pm 1$

$$
k_{ \pm}=\frac{1}{\sqrt{\pi}} \int_{-1}^{1} \sqrt{\frac{1 \pm s}{1 \mp s}} g(s) d s
$$

Requiring that this intensity factor vanish at both tips, hence implies that two conditions be 
satisfied by the distribution $g(s)$

$$
\int_{-1}^{1} \sqrt{\frac{1 \pm s}{1 \mp s}} g(s) d s=0
$$

which can be recast as the sum and the difference of these two conditions, leading respectively to

$$
\int_{-1}^{1} \frac{g(s)}{\sqrt{1-s^{2}}} d s=0 \quad \int_{-1}^{1} \frac{s g(s)}{\sqrt{1-s^{2}}} d s=0
$$

In the problem for the slip distribution, eq. (4.1), we identify

$$
g(s)=\operatorname{erfc}|\lambda s|-\left(1-\frac{\tau}{\tau_{p}}\right) \frac{\sigma^{\prime}}{\Delta p}
$$

The first condition of (A 7) to be satisfied by (A 8) corresponds to eq. (4.2) in main text, which provides the direct relation between $\lambda$ and the problem parameter $\left(1-\tau / \tau_{p}\right) \sigma^{\prime} / \Delta p$. The second condition of (A 7) is trivially satisfied by (A 8), since $g(s)$ is an even function for that case.

The non-singular stress conditions (A 7), if present, can be taken into account in the inversion for $h^{\prime}(x)$, eq. (A 2), which may be rewritten as

$$
\begin{aligned}
h^{\prime}(x) & =-\frac{1}{\sqrt{1-x^{2}}} \frac{1}{\pi} \int_{-1}^{1} \frac{\sqrt{1-s^{2}} g(s)}{x-s} d s+\frac{1}{\sqrt{1-x^{2}}} \frac{1}{\pi} \int_{-1}^{1} \frac{(x+s) g(s)}{\sqrt{1-s^{2}}} d s \\
& =-\frac{\sqrt{1-x^{2}}}{\pi} \int_{-1}^{1} \frac{1}{\sqrt{1-s^{2}}} \frac{g(s)}{x-s} d s
\end{aligned}
$$

Upon substituting eq. (A 8) in eq. (A 9), the contribution of the constant parameter (1 $\left.\tau / \tau_{p}\right) \sigma^{\prime} / \Delta p$ vanishes, and we retrieve eq. (4.3) in the main text.

As another example, we again consider the distribution $g(x)=\delta_{D}(x)$, for which the inversion for $h^{\prime}(x)$ with the non-singular condition on $h^{\prime}(x)$ is

$$
\begin{gathered}
h^{\prime}(x)=\frac{-\sqrt{1-x^{2}}}{\pi} \int_{-1}^{1} \frac{1}{\sqrt{1-s^{2}}} \frac{\delta_{D}(s)}{x-s} d s=-\frac{1}{\pi} \frac{\sqrt{1-x^{2}}}{x}=-\frac{1}{\pi}\left(\frac{1}{x \sqrt{1-x^{2}}}-\frac{x}{\sqrt{1-x^{2}}}\right) \\
h(x)=\frac{1}{\pi}\left(\operatorname{arctanh} \sqrt{1-x^{2}}-\sqrt{1-x^{2}}\right)
\end{gathered}
$$

to which we may compare, eq. (A 4), the inversion for the same distribution $g(x)$ without the non-singular condition.

In writing an asymptotic expansion for slip in powers $\lambda$ in the marginally pressurized and critically stressed limits, we derived spatial distributions for slip at each order $\left(\delta_{0}\right.$ and $\left.\delta_{1}\right)$ using results presented in Table 1 . Note that the compilation of inversions in Table 1 used (A 2) and did not incorporate the non-singular crack conditions (A 7) in the inversion for $h^{\prime}(x)$ from $g(x)$. However, when solving for $\delta_{0}$ and $\delta_{1}$, the non-singular conditions are implicitly incorporated in the expansion for the stress parameter $\left(1-\tau / \tau_{p}\right) \sigma^{\prime} / \Delta p$ in terms of $\lambda$. The resulting expressions for $\delta_{0}$ and $\delta_{1}$ are fully equivalent to the solutions that would have been found had the conditions (A 7) been incorporated directly into the inversion. In other words, we would have arrived to the same expressions for $\delta_{0}$ and $\delta_{1}$ in Sections 5 and 6 had we instead used the non-singular inversion (A 10) to construct the functions $h(x)$ in Table 1 and subsequently applied them to solve for $\delta_{0}$ and $\delta_{1}$. We recognize this, for instance, 
in noting that the first term in the expansion for slip in the critically-stressed limit, eq. (6.9), is given, to within the factor $p_{0}=2 / \sqrt{\pi}$ by eq. (A 10 ).

\section{Appendix B. Numerical solution procedure}

To numerically solve for the slip distribution $\bar{\delta}(\bar{x})$ from eq. (4.3), given a value of $\lambda$, we use Gauss-Chebyshev quadrature for singular integrals (Erdogan et al. 1973; Viesca and Garagash 2018). We begin by separating the singular integral in eq. (4.3)

$$
\phi(\bar{z})=-\frac{1}{\pi} \int_{-1}^{1} \frac{\operatorname{erfc}|\lambda \bar{s}|}{\sqrt{1-\bar{s}^{2}}} \frac{1}{\bar{z}-\bar{s}} d s
$$

and note that the slip distribution is given by the integration

$$
\bar{\delta}(\bar{x})=\int_{-1}^{\bar{x}} \sqrt{1-\bar{z}^{2}} \phi(\bar{z}) d \bar{z}
$$

We numerically approximate the integral (B 1) at a discrete set of points $z_{i}$

$$
\phi\left(\bar{z}_{i}\right) \approx-\frac{1}{n} \sum_{j=1}^{n} \frac{\operatorname{erfc}\left|\lambda \bar{s}_{j}\right|}{\bar{z}_{i}-\bar{s}_{j}}
$$

where the quadrature points $\bar{s}_{j}$ and $\bar{z}_{i}$ are

$$
\begin{aligned}
& \bar{z}_{i}=\cos (\pi i / n) \quad i=1, \ldots, n-1 \\
& \bar{s}_{j}=\cos [\pi(j-1 / 2) / n] \quad j=1, \ldots, n
\end{aligned}
$$

We again use Gauss-Chebyshev quadrature (Mason and Handscomb 2002) to approximate the integral (B 2) at a set of points $x_{k}$

$$
\bar{\delta}\left(\bar{x}_{k}\right) \approx \frac{1}{n} \sum_{i=1}^{n-1}\left(1-\bar{z}_{i}^{2}\right) \phi\left(\bar{z}_{i}\right) H\left(\bar{x}_{k}-\bar{z}_{i}\right)
$$

where $\bar{x}_{k}=\bar{s}_{k}$ for $k=1, \ldots, n$ and $H(\cdot)$ is the Heaviside step function.

We may similarly approximate the relation between $\left(1-\tau / \tau_{p}\right) \sigma^{\prime} / \Delta p$ and $\lambda$ by first prescribing a value of $\lambda$ and then numerically evaluating the integral (4.2) by GaussChebyshev quadrature

$$
\left(1-\frac{\tau}{\tau_{p}}\right) \frac{\sigma^{\prime}}{\Delta p} \approx \frac{1}{n} \sum_{j=1}^{n} \operatorname{erfc}\left|\lambda s_{j}\right|
$$

where $s_{j}$ is defined as before.

\section{Appendix C. Multipole expansion}

Here we derive the multipole expansion of eqs. (6.3-6.6). This expansion was used to derive the outer solution in the critically stressed limit, for which the rupture extent $a(t) \gg \sqrt{\alpha t}$, such that the fluid pressure source appears localized about the origin. We begin by noting that the solution to the problem for $h(x)$

$$
g(x)=\frac{1}{\pi} \int_{-1}^{1} \frac{h^{\prime}(s)}{x-s} d s
$$


with the boundary conditions $h(-1)=h(1)=0$, may be also written in terms of the Green's function $G\left(x, x^{\prime}\right)$ satisfying

$$
\delta_{D}\left(x-x^{\prime}\right)=\frac{1}{\pi} \int_{-1}^{1} \frac{G\left(s, x^{\prime}\right)}{x-s} d s
$$

Using the inversion eq. (A 2), the Green's function is

$$
\begin{aligned}
G\left(x, x^{\prime}\right) & =-\frac{1}{\sqrt{1-x^{2}}} \frac{1}{\pi} \int_{-1}^{1} \frac{\sqrt{1-s^{2}} \delta_{D}\left(s-x^{\prime}\right)}{x-s} d s \\
& =-\frac{1}{\pi} \sqrt{\frac{1-x^{\prime 2}}{1-x^{2}}} \frac{1}{x-x^{\prime}}
\end{aligned}
$$

and the solution to eq. (C 1) can be written as

$$
h^{\prime}(x)=\int_{-1}^{1} G\left(x, x^{\prime}\right) g\left(x^{\prime}\right) d x^{\prime}
$$

We derive a multipole expansion for eq. (C 1) by considering that a Taylor series expansion of the Green's function in eq. (C 3) about the origin $x^{\prime}=0$

$$
h^{\prime}(x)=\int_{-1}^{1}\left[G(x, 0)+\left.x^{\prime} \frac{\partial G}{\partial x^{\prime}}\right|_{x^{\prime}=0}+\left.\frac{x^{\prime 2}}{2} \frac{\partial^{2} G}{\partial x^{\prime 2}}\right|_{x^{\prime}=0}+\ldots+\left.\frac{x^{\prime n}}{n !} \frac{\partial^{n} G}{\partial x^{\prime n}}\right|_{x^{\prime}=0}\right] g\left(x^{\prime}\right) d x^{\prime}
$$

reduces to

$$
h^{\prime}(x)=p_{0} G(x, 0)+\left.p_{1} \frac{\partial G}{\partial x^{\prime}}\right|_{x^{\prime}=0}+\left.p_{2} \frac{\partial^{2} G}{\partial x^{\prime 2}}\right|_{x^{\prime}=0}+\ldots+\left.p_{n} \frac{\partial^{n} G}{\partial x^{\prime n}}\right|_{x^{\prime}=0}
$$

where the coefficients of this series are

$$
\begin{aligned}
& p_{0}=\int_{-1}^{1} g\left(x^{\prime}\right) d x^{\prime}, \quad p_{1}=\int_{-1}^{1} x^{\prime} g\left(x^{\prime}\right) d x^{\prime}, \\
& p_{2}=\int_{-1}^{1} \frac{x^{\prime 2}}{2} g\left(x^{\prime}\right) d x^{\prime}, \quad \ldots, \quad p_{n}=\int_{-1}^{1} \frac{x^{\prime n}}{n !} g\left(x^{\prime}\right) d x^{\prime}
\end{aligned}
$$

The expansion of $g(x)$ implied by eq. (C 4$)$ is found by first noting that, from eq. (C 2),

$$
\frac{\partial^{n} G}{\partial x^{\prime n}}=-\frac{1}{\sqrt{1-x^{2}}} \frac{1}{\pi} \int_{-1}^{1} \frac{\sqrt{1-s^{2}}\left[(-1)^{n} \delta_{D}^{(n)}\left(s-x^{\prime}\right)\right]}{x-s} d s
$$

where $\delta_{D}^{(n)}$ is the $n$-th derivative of $\delta_{D}$ with respect to its argument. When evaluating eq. (C 5) at $x^{\prime}=0$ and comparing with eq. (A 2), we see that eq. (C 5) is the inverted solution for $h^{\prime}(x)$ when $g(x)=(-1)^{n} \delta_{D}^{(n)}(x)$, hence substituting eq. (C 4) into eq. (C 1) yields

$$
g(x)=p_{0} \delta_{D}(x)-p_{1} \delta_{D}^{\prime}(x)+p_{2} \delta_{D}^{\prime \prime}(x)-\ldots+(-1)^{n} p_{n} \delta_{D}^{(n)}(x)
$$

where the leading two terms are the source monopole and dipole approximations, respectively. For $n=0,1,2$, the first few functions of $x$ in eq. (C 4) are

$$
G(x, 0)=-\frac{1}{\pi} \frac{1}{x \sqrt{1-x^{2}}},\left.\quad \frac{\partial G}{\partial x^{\prime}}\right|_{x^{\prime}=0}=-\frac{1}{\pi} \frac{1}{x^{2} \sqrt{1-x^{2}}},\left.\quad \frac{\partial^{2} G}{\partial x^{\prime 2}}\right|_{x^{\prime}=0}=\frac{x^{2}-2}{x^{3} \sqrt{1-x^{2}}}
$$

Multiplying these functions by $(-1)^{n}$ and integrating with respect to $x$, we find the expressions in Table 1 for $h(x)$ when $g(x)=\delta_{D}(x)$ or one of its first two derivatives. 


\section{REFERENCES}

Adachi, J. I., And Detournay, E. 2008 Plane strain propagation of a hydraulic fracture in a permeable rock, Eng. Frac. Mech., 75, pp. 4666-4694.

Ball, T. V. And Neufeld, J. A. 2018 Static and dynamic fluid-driven fracturing of adhered elastica, Phys. Rev. Fluids, 3, 074101.

BARENBLATt, G. I. 1956 On the formation of horizontal cracks in hydraulic fracture of an oil-bearing stratum, Prikl. Mat. Mech., 20, pp. 475-486.

Bhattacharya, P., And Viesca, R. C. 2019 Fluid-induced aseismic fault slip outpaces pore-fluid migration, Science, 364, pp. 464-468.

BRANTUT, N. 2021 Dilatancy toughening of shear cracks and implications for slow rupture propagation, arXiv, 2104.06475.

Bunger, A. P., And Cruden, A. R. 2011 Modeling the growth of laccoliths and large mafic sills: role of magma body forces, J. Geophys. Res., 116, B02203.

Ciardo, F., and Lecampion, B. 2019 Effect of dilatancy on the transition from aseismic to seismic slip due to fluid injection in a fault, J. Geophys. Res., 124, pp. 3724-3743.

Desroches, J., Detournay, E., Lenoach, B., Papanastasiou, P., Pearson, J. R. A., M. Thiercelin, and Cheng, A. 1994 The crack tip region in hydraulic fracturing, Proc. Roy. Soc. Lond. A, 447, pp. 39-48.

Detournay, E. 2016 Mechanics of hydraulic fractures, Annu. Rev. Fluid Mech., 48, pp. 311-339.

Dublanchet, P. 2019 Fluid driven shear cracks on a strengthening rate-and-state frictional fault, J. Mech. Phys. Solids, 132, 103672.

Erdogan F., Gupta, G.D., and Cook, T.S. 1973 Numerical solution of singular integral equations. Ch. 7 from Methods of Analysis and Solutions of Crack Problems, (ed. Sih, G.C.), Mechanics of Fracture, vol. 1, pp. 368-425, Springer.

Flitton, J. C., AND King, J. R. 2004 Moving boundary and fixed-domain problems for a sixth-order thin-film equation, Euro. J. Appl. Math., 15, pp. 713-754.

Garagash, D. I. 2012 Seismic and aseismic slip pulses driven by thermal pressurization of pore fluid, $J$. Geophys. Res., 117, B04314.

Garagash, D. I. 2021 Fracture mechanics of rate-and-state faults and fluid injection induced slip, Phil. Trans. Roy. Soc. A, 379, 20200129.

Garagash, D. I., and Detournay, E. 2000 The tip region of a fluid-driven fracture in an elastic medium, J. Appl. Mech., 67, pp. 183-192.

Garagash, D. I., and Germanovich, L. N. 2012 Nucleation and arrest of dynamic slip on a pressurized fault, J. Geophys. Res., 117, B10310.

Garipov, T. T., Karimi-Fard, M., and Tchelepi, H. A. 2016 Discrete fracture model for coupled flow and geomechanics, Comput. Geosci., 20, pp. 149-160.

Healy, J. H., Rubey, W. W., Griggs, D. R., and Raleigh, C. B. 1966 The Denver earthquakes, Science, 161, pp. 1301-1310.

Hewitt, I. J., Balmforth, N. J., And de Bruyn, J. R. 2015 Elastic-plated gravity currents, Euro. J. Appl. Math., 26, pp. 1-31.

Hinch, E. J. 1991 Perturbation Methods, Cambridge University Press.

Hosoi, A. E., And Mahadevan, L. 2004 Peeling, healing, and bursting in a lubricated elastic sheet, Phys. Rev. Lett., 93, 137802.

JhA, B., AND JuAnes, R. 2014 Coupled multiphase flow and poromechanics: A computational model of pore pressure effects on fault slip and earthquake triggering, Wat. Resour. Res., 50, pp. 3776-3808.

Khristianovic, S. A., And Zheltov, Y. P. 1955 Formation of vertical fractures by means of highly viscous fluid, Proc. 4th World Petrol. Congr., 1, pp. 579-586, World Petroleum Council.

Kennedy, B. M., Kharaka, Y. K., Evans, W. C., Ellwood, A., DePaolo, D. J., Thordsen, J., Ambats, G., And Mariner, R. H. 1997 Mantle fluids in the San Andreas fault system, California, Science., 278, pp. 1278-1281.

KInG, F. W. 2009 Hilbert Transforms, vols. 1 \& 2, Cambridge University Press.

LeNOACH, B. 1995 The crack tip solution for hydraulic fracturing in a permeable solid, J. Mech. Phys. Solids, 43, pp. 1025-1043.

Lipovsky, B. P., And Dunham, E. R. 2017 Slow-slip events on the Whillans Ice PLain, Antarctica, described using rate-and-state friction as an ice stream sliding law, J. Geophys. Res., 122, pp. 973-1003. 
ListeR, J. R. 1990 Buoyancy-driven fluid fracture: the effects of material toughness and of low-viscosity precursors, J. Fluid Mech., 210, pp. 263-280.

ListeR, J. R. 1990 Buoyancy-driven fluid fracture: similarity solutions for the horizontal and vertical propagation of fluid-filled cracks, J. Fluid Mech., 217, pp. 213-239.

Lister, J. R., Peng, G. G., And Neufeld, J. A. 2013 Viscous control of peeling an elastic sheet by bending and pulling, Phys. Rev. Lett., 111, 154501.

Lister, J. R., Skinner, D. J., and Large, T. M. J. 2019 Viscous control of shallow elastic fracture: peeling without precursors, J. Fluid. Mech., 868, pp. 119-140.

Marck, J., Savitski, A. A., and Detournay, E. 2015 Line source in a poroelastic layer bounded by an elastic space, Int. J. Numer. Anal. Meth. Geomech., 39, pp. 1484-1505.

Mason, J. C., And Handscomb, D. C. 2002 Chebyshev Polynomials, Chapman and Hall.

McClung, D. M. 1979 Shear fracture precipitated by strain softening as a mechanism, J. Geophys. Res., 84, pp. 3519-3526.

Michaut, C. M. 2011 Dynamics of magmatic intrusions in the upper crust: theory and applications to laccoliths on Earth and the Moon, J. Geophys. Res., 116, B05205.

Mushrhelishvili, N. I. 1958 Singular Integral Equations, Springer.

Noda, H., Dunham, E. M., And Rice, J. R. 2009 Earthquake ruptures with thermal weakening and the operation of major faults at low overall stress levels, J. Geophys. Res., 114, pp. B07302.

Palmer, A. C., ANd Rice, J. R. 1973 The growth of slip surfaces in the progressive failure of over-consolidated clay, Proc. R. Soc. Lon. A, 332, pp. 527-548.

Peacock, S. M. 2001 Are the lower planes of double seismic zones caused by serpentine dehydration in subducting oceanic mantle?, Geology, 29, pp. 299-302.

Peng, G. G., And Lister, J. R. 2020 Viscous flow under an elastic sheet, J. Fluid Mech., 905, A30.

Platt, J. D., Viesca, R. C., and Garagash, D. I. 2015 Steadily propagating slip pulses driven by thermal decomposition, J. Geophys. Res., 120, pp. 6558-6591.

Puzrin, A. M., and Germanovich, L. N. 2005 The growth of shear bands in the catastrophic failure of soils, Proc. R. Soc. A, 461, pp. 1199-1228.

Rice J. R. 1968 Mathematical analysis in the mechanics of fracture. Ch. 3 from Fracture, vol. 2, (ed. Liebowitz, H.), pp. 191-311, Academic Press.

Rice J. R. 1973 The initiation and growth of shear bands Proceedings of the Symposium on the Role of Plasticity in Soil Mechanics, vol. 2, (ed. Palmer, A. C.), pp. 263-274, Cambridge University Department of Engineering.

Rice, J. R. 2006 Heating and weakening of faults during earthquake slip, J. Geophys. Res., 111, B05311.

Rubin, A. M. 1995 Propagation of magma-filled cracks, Annu. Rev. Earth Planet. Sci., 23, pp. 287-336.

RutQvist, J., Birkholzer, J., CAPPA, F., AND Tsang, C.-F. 2007 Estimating maximum sustainable injection pressure during geological sequestration of $\mathrm{CO} 2$ using coupled fluid flow and geomechanical faultslip analysis, Energ. Convers. Manage., 48, pp. 1798-1807.

Schmitt, S. V., Segall, P., and Matsuzawa, T. 2011 Shear heating-induced thermal pressurization during earthquake nucleation, J. Geophys. Res., 116, B06308.

Spence, D. A., And Sharp, P. 1985 Self-similar solutions for elastohydrodynamic cavity flow, Proc. R. Soc. Lond. A, 400, pp. 289-313.

Tsai, V. C., And Rice, J. R. 2010 A model for turbulent hydraulic fracture and application to crack propagation at glacier beds, J. Geophys. Res., 114, F03007.

Wang, Z.-Q. and Detournay, E. 2018 The tip region of a near-surface hydraulic fracture, J. Appl. Mech., 85, 041010.

Williams, M. L. 1957 On the stress distribution at the base of a stationary crack, J. Appl. Mech., 79, pp. $109-114$.

Viesca, R. C., And Garagash, D. I. 2015 Ubiquitous weakening of faults due to thermal pressurization, Nat. Geosci., 8, pp. 875-879.

Viesca, R. C., And Garagash, D. I. 2018 Numerical methods for coupled fracture problems, J. Mech. Phys. Solids, 113, pp. 13-34.

ViesCA, R. C., AND Rice, J. R. 2012 Nucleation of slip-weakeing rupture instability in landslides by localized increase of pore pressure, J. Geophys. Res., 117, B03104.

YAnG, Y. And Dunham, E. M. 2021 Effect of porosity and permeability evolution on injection-induced aseismic slip, J. Geophys. Res., 126, e2020JB021258. 
Zhu, W., Allison, K. L., Dunham, E. M., And Yang, Y. 2020 Fault valving and pore pressure evolution in simulations of earthquake sequences and aseismic slip, Nat. Comm., 11, 4833. 